\section{(6) OPEN ACCESS}

\title{
Apelin targets gut contraction to control glucose metabolism via the brain
}

\author{
Audren Fournel, ${ }^{1,2,3}$ Anne Drougard, ${ }^{1,2,3}$ Thibaut Duparc, ${ }^{2,4}$ Alysson Marlin, ${ }^{1,2,3}$ \\ Stuart M Brierley, ${ }^{5,6,7}$ Joel Castro, ${ }^{5}$ Sophie Le-Gonidec, ${ }^{1,2,3}$ Bernard Masri, ${ }^{8}$ \\ André Colom, ${ }^{1,2,3}$ Alexandre Lucas, ${ }^{1,2,3}$ Perrine Rousset, ${ }^{3,9}$ Nicolas Cenac, $^{3,9}$ \\ Nathalie Vergnolle, ${ }^{3,9}$ Philippe Valet, ${ }^{1,2,3}$ Patrice D Cani, ${ }^{2,4}$ Claude Knauf ${ }^{1,2,3}$
}

\begin{abstract}
- Additional material is published online only. To view please visit the journal online (http://dx.doi.org/10.1136/ gutjnl-2015-310230)

For numbered affiliations see end of article.
\end{abstract}

\section{Correspondence to} Professor Patrice D Cani, Université Catholique de Louvain (UCL), Louvain Drug Research Institute, LDRI, Metabolism and Nutrition research group, WELBIO, WELBIO (Walloon Excellence in Life sciences and BIOtechnology), Av. E. Mounier, 73 B1.73.11, Brussels B-1200, Belgium: Patrice.cani@uclouvain.be Professor Claude Knauf, Institut National de la Santé et de la Recherche Médicale (INSERM), U1048, Institut des Maladies Métaboliques et Cardiovasculaires (I2MC), Université Paul Sabatier, CHU Rangueil, 1 Avenue Jean Poulhès, BP84225, 31432 Toulouse Cedex 4, France; Claude.knauf@inserm.fr

Received 24 June 2015 Revised 2 September 2015 Accepted 22 September 2015 Published Online First 12 November 2015
CrossMark

To cite: Fournel $A$ Drougard A, Duparc T, et al. Gut 2017:66:258-269.

\section{ABSTRACT}

Objective The gut-brain axis is considered as a major regulatory checkpoint in the control of glucose

homeostasis. The detection of nutrients and/or hormones in the duodenum informs the hypothalamus of the host's nutritional state. This process may occur via

hypothalamic neurons modulating central release of nitric oxide (NO), which in turn controls glucose entry into tissues. The enteric nervous system (ENS) modulates intestinal contractions in response to various stimuli, but the importance of this interaction in the control of glucose homeostasis via the brain is unknown. We studied whether apelin, a bioactive peptide present in the gut, regulates ENS-evoked contractions, thereby identifying a new physiological partner in the control of glucose utilisation via the hypothalamus.

Design We measured the effect of apelin on electrical and mechanical duodenal responses via telemetry probes and isotonic sensors in normal and obese/diabetic mice. Changes in hypothalamic NO release, in response to duodenal contraction modulated by apelin, were evaluated in real time with specific amperometric probes. Glucose utilisation in tissues was measured with orally administrated radiolabeled glucose.

Results In normal and obese/diabetic mice, glucose utilisation is improved by the decrease of ENS/contraction activities in response to apelin, which generates an increase in hypothalamic NO release. As a consequence, glucose entry is significantly increased in the muscle.

Conclusions Here, we identify a novel mode of communication between the intestine and the hypothalamus that controls glucose utilisation. Moreover, our data identified oral apelin administration as a novel potential target to treat metabolic disorders.

\section{INTRODUCTION}

The gut-brain axis is of crucial importance in the control of energy homeostasis. The detection or 'sensing' of intestinal lipids ${ }^{1}$ and glucose ${ }^{2}$ activates extrinsic afferent nerves in the gut wall, which inform the hypothalamus about the presence of nutrients in the digestive tract. The subsequent modulation of hypothalamic neuronal activity in response to these peripheral signals leads to modification of metabolic functions including thermogenesis, ${ }^{3}$ food intake and glucose utilisation in tissues. ${ }^{4}$

Recent studies demonstrate the importance of gut nutrient sensing in the control of glucose

\section{Significance of this study}

What is already known on this subject?

- Circulating apelin is a bioactive peptide that exerts pleiotropic actions in various organs (brain, muscle, adipose tissue) to control glucose utilisation and glycaemia.

- The gut-to-brain axis is of crucial importance in the control of glucose homeostasis and is profoundly altered during metabolic diseases such as type 2 diabetes.

- Enteric nervous system (ENS) is under the influence of various bioactive factors such as leptin, which is able to be transcytosed from the lumen to the gut wall.

\section{What are the new findings?}

- Luminal apelin is able to be transcytosed through the intestine to reach intraduodenal structures.

- Apelin controls ENS neurotransmitter release, that is, acetylcholine and nitric oxide, associated to variations of duodenal contraction.

- Apelin triggers ENS-induced duodenal contraction, leading to muscle glucose absorption via hypothalamic relay.

- Chronic oral administration of apelin improves glucose tolerance in closed correlation to a decrease in duodenal motility in normal and obese/diabetic mice.

How might it impact on clinical practice in the foreseeable future?

- This study provides unequivocal evidences that modulation of the ENS/contraction of the duodenum is a new physiological system controlling peripheral glucose utilisation via the brain. More importantly, oral apelin administration could be considered as a promising therapeutic target to treat insulin resistance state.

homeostasis. ${ }^{5}$ Alteration of nutrient detection in the duodenum and/or jejunum ${ }^{6}$ disturbs hypothalamic responses that could contribute to the establishment of increased hepatic glucose production ${ }^{7}$ 
and insulin resistance, ${ }^{2}$ characteristic features of type 2 diabetes. The identification of new molecular mechanisms that are able to modify the gut-brain axis is of major significance in discovering new and successful therapeutic strategies. For example, leptin, which is secreted in the luminal part of the intestine in response to nutrients, can modify the activity of phosphatidylinositol 3 kinase in the jejunum to decrease hepatic glucose production. ${ }^{7}$ In addition to leptin, apelin is another potential target that exerts pleiotropic effects in the whole body. Notably, apelin is recognised as a bioactive peptide implicated in the control of glucose metabolism. ${ }^{8}$ In the central nervous system (CNS), apelin is a neurotransmitter able to target hypothalamic neurons to control glycaemia. ${ }^{9}$ In the periphery, apelin is an adipokine that improves insulin sensitivity of muscles from normal and obese/diabetic mice. ${ }^{8}{ }^{10}$ Recent data show that apelin is also present and released in the luminal part of the intestine to stimulate glucose absorption. ${ }^{11}$

In the GI tract, the enteric nervous system (ENS) is involved in various physiological functions including modulation of intestinal contraction. The ENS is essentially composed of excitatory (choline acetyl transferase (ChAT)) motor neurons or inhibitory motor neurons (neuronal nitric oxide synthase (nNOS)) that stimulate or inhibit intestinal contractility and motility. ${ }^{12}$ These ENS neurons are known to express receptors for various factors such as leptin ${ }^{13}$ and glucagon-like peptide-2 (GLP-2). ${ }^{14}$ In the later stage of diabetes, alterations of ENS in the proximal part of the intestine (which is being affected first) are associated with an intestinal hypercontractility. ${ }^{12}$ Furthermore, several studies have demonstrated that during diabetes there is a loss of inhibitory neurons and an increase in cholinergic innervations in the proximal part of the intestine. ${ }^{12}$ Whether the duodenum contraction-controlled by ENS is implicated in the control of glucose homeostasis via the brain is totally unknown.

Here, we investigate whether GI apelin can modify the activity of the 'ENS/contraction' couple to control whole-body glucose utilisation via the brain. Overall, our study unravels a new mode of communication involving the mechanical contractility of the duodenum, which could be considered as a potential therapeutic target to improve tissue glucose utilisation via the brain during type 2 diabetes.

\section{MATERIALS AND METHODS}

\section{Mice}

Male C57BL/6J mice (Charles River Laboratory, l'Arbresle, France) had free access to food and water. Mice were fed on normal chow or high-fat diet (HFD) containing 20\% protein, $35 \%$ carbohydrate and $45 \%$ fat (Research Diets, New Brunswick, New Jersey, USA). These mice became obese and insulin resistant after 3 months of HFD. ${ }^{9}$ Data have been recorded in fed mice, corresponding to the phase where intestinal segmental waves are generated to increase the rate of nutrients absorption. ${ }^{15}$

Acute injections (apelin, apelin receptor (APJ) antagonist, NOS inhibitor, agonist of $\boldsymbol{\beta}$-adrenergic receptors, antagonist of nicotinic receptors and capsaicin)

Protocol are described in online supplementary methods section.

\section{Chronic administration of apelin}

In other experimental sets of mice, $100 \mu \mathrm{L}$ of [Pyr]apelin-13 at a concentration of $1 \mu \mathrm{M}$ or $100 \mu \mathrm{L}$ of $\mathrm{H}_{2} \mathrm{O}$ were orally loaded (oral gavage), once a day for 1 week.
Intragastric and intracerebroventricular surgical procedures, telemetry, isotonic contractions, immunohistochemistry, transcytosis, acetylcholine release, glucose and apelin absorption, glucose utilisation, qPCR, metabolic parameters, insulin and apelin assays, oral glucose tolerance test Protocols are described in online supplementary methods section.

\section{Cell culture and fluorescence microscopy}

HEK-293T cells were cultured as previously described ${ }^{16}$ to test the functionality of apelin-TAMRA. Protocol is described in detail in online supplementary methods section. Apelin-TAMRA is a functional apelin molecule that is able to bind and activate its receptor APJ (see online supplementary figure S1).

\section{Ex vivo duodenal real-time NO measurement}

Mice were euthanased in fed conditions. After dissection, duodenum fragments were washed in Krebs-Ringer bicarbonate/ glucose buffer $(\mathrm{pH} 7.4)$ in an atmosphere of $95 \% \mathrm{O}_{2}-5 \% \mathrm{CO}_{2}$ and then immersed in Eppendorf tubes containing $400 \mu \mathrm{L}$ of the same medium. After a $10 \mathrm{~min}$ recovery period, the spontaneous $\mathrm{NO}$ release was measured at $37^{\circ} \mathrm{C}$ for $20 \mathrm{~min}$ by using an NO-specific amperometric probe (ISO-NOPF, $100 \mu \mathrm{m}$ diameter, $5 \mathrm{~mm}$ length, World Precision Instruments, Aston Stevenage, UK) implanted directly in the duodenum. Data are expressed as the change (delta) in NO release from basal conditions. ${ }^{9}$

\section{In vivo hypothalamic real-time NO measurement}

Mice in fed conditions were anesthetised with isoflurane. A $1 \mathrm{~cm}$ midline incision was made across the top of the skull, and the animal was placed on a stereotaxic apparatus, as described previously. ${ }^{6}$ Results are presented as mean \pm SEM. During amperometric measurement, animals received $\mathrm{H}_{2} \mathrm{O}$ or drugs via an intragastric injection of $10 \mu \mathrm{L} / \mathrm{min}$ rate for $10 \mathrm{~min}$ using a syringe pump. We previously demonstrated that $\mathrm{H}_{2} \mathrm{O}$ is the vehicle of choice for glucose administration. ${ }^{2} 617$ Furthermore, we have also demonstrated that intragastric perfusion of $\mathrm{H}_{2} \mathrm{O}$ did not modify c-Fos expression (a marker of neuronal activity) in the hypothalamus compared with $\mathrm{NaCl}(0.9 \%)$. Therefore, all control groups were infused with water, as previously described in detail. ${ }^{2}$

\section{Statistical analysis}

The data are expressed as mean \pm SEM. Differences between the experimental groups were assessed where appropriate using unpaired Student's and one-way or two-way analysis of variance, followed by post hoc Bonferroni test. Data were analysed using GraphPad Prism V.5.00 for Windows (GraphPad Software, San Diego, California, USA). The results were considered statistically significant at $\mathrm{p}<0.05$.

\section{RESULTS}

\section{Apelin acts on ENS neurons to control duodenal contractions}

The duodenum is the first intestinal site of nutrient sensing ${ }^{5}$ and apelin's effect on glucose absorption. ${ }^{11}$ Using immunohistochemistry, we found that the apelin receptor APJ is expressed on ChAT and nNOS neurons (figure 1A). To determine whether apelin is able to undergo transcytosis through the intestine, we used apelin-TAMRA, a functional apelin molecule able to bind to the apelin receptor APJ (see online supplementary figure S1). We observed that apelin is able to reach underlying structures 


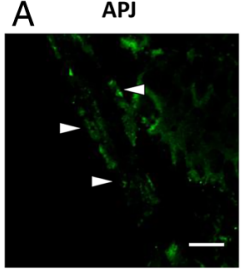

APJ

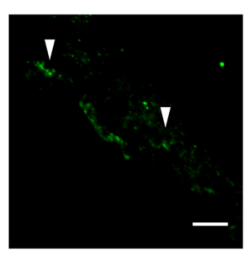

B

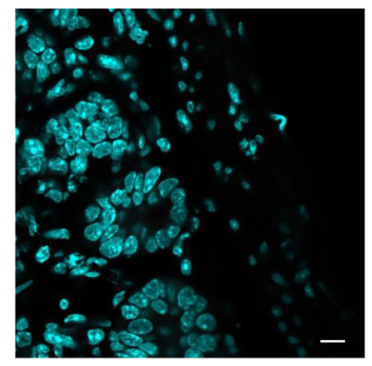

ChAT

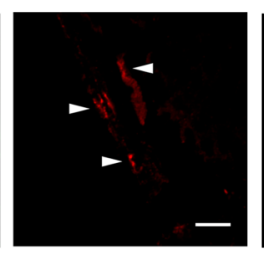

nNOS

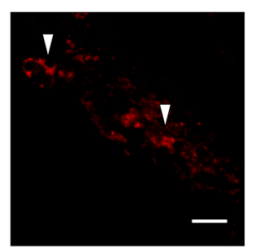

APJ/ChAT

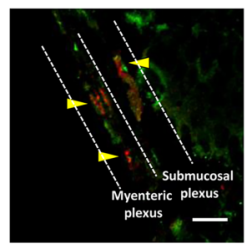

$\mathrm{APJ} / \mathrm{nNOS}$
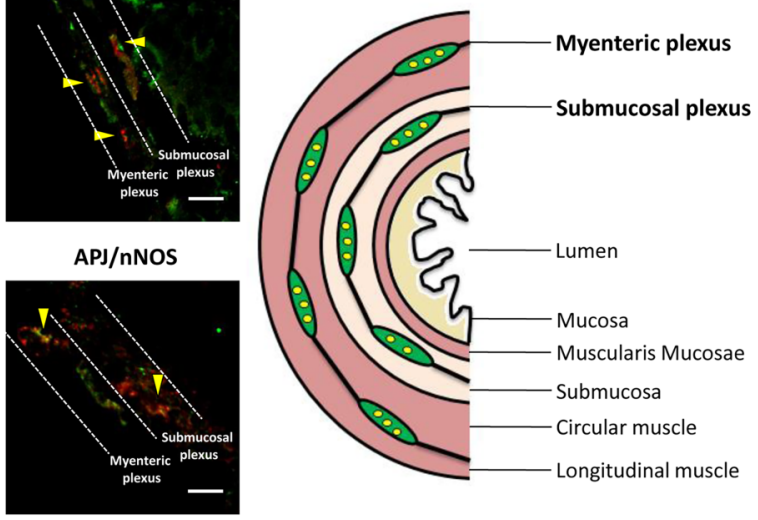

Figure 1 Apelin receptor (APJ) is expressed in enteric nervous system neurons. (A) Duodenal sections from mice stained with anti-APJ antibody (left panels, green: white arrows), anti-choline acetyl transferase (ChAT) or anti-neuronal nitric oxide synthase (nNOS) antibodies (middle panels, red: white arrows) and merge (right panels, yellow arrows). Pictures are representative of four mice per groups. Bars=10 $\mu \mathrm{m}$. Right panel: schematic representation of a transverse section through the duodenum. (B) Duodenal sections from mice perfused for 5 min with $\mathrm{H}_{2} \mathrm{O}$ (Control) (left panel) or apelin-TAMRA (right panel). Apelin-TAMRA appears in red (red arrows). Pictures are representative of three mice per groups. Bars $=10 \mu \mathrm{m}$.

such as neuronal plexus (figure 1B), suggesting apelin, like other adipokines such as leptin, ${ }^{18}$ undergo transcytosis.

To assess whether apelin is able to control duodenal motility via the ENS, we used isotonic sensors to measure ex vivo mechanical contractions (see online supplementary figure S2A). Validation of the technique was obtained by using L-NG-nitroarginine methyl ester (L-NMMA) (an inhibitor of NOS enzymes) and isoproterenol (an adrenergic receptor agonist), which stimulated ${ }^{19}$ and inhibited ${ }^{20}$ intestinal contractions, respectively (see online supplementary figure S2B). Application of apelin on ex vivo duodenal preparations increased the amplitude of intestinal contractions $(10 \mathrm{pM}$ to $100 \mathrm{nM}$ ) (figure 2A) but did not affect the frequency of contractions (see online supplementary figure S2C). The effects of apelin are specific to the duodenum since apelin did not modulate jejunum and ileum contractions (see online supplementary figure S2D). However, concentrations of apelin $>1 \mu \mathrm{M}$ generated an amplitude of contraction profile similar to that observed at basal levels, suggesting the existence of a physiological switch from a stimulatory effect $(10 \mathrm{pM}$ to $100 \mathrm{nM})$ to basal and/or inhibitory state $(1-10 \mu \mathrm{M})$ (figure $2 \mathrm{~A})$. This last result suggested the existence of two different signalling pathways for the two doses, and all these effects are antagonised by F13A (see online supplementary figure S2E), an APJ receptor antagonist. To this aim, we choose to focus our intention on the closest doses (ie, $100 \mathrm{nM}$ and $1 \mu \mathrm{M})$ implicated in this physiological switch. Stimulatory effect of apelin on intestinal contractions needed ChAT neurons since hexamethonium, a nicotinic receptor antagonist, blocked its effect (figure 2B). Moreover, stimulation of duodenal contraction by apelin $100 \mathrm{nM}$ is associated with an increase of duodenal acetylcholine release (figure 2C) without dose-effect (see online supplementary figure S3A), whereas NO is not affected following the exposure to $100 \mathrm{nM}$ of apelin (figure 2D). Apelin $1 \mu \mathrm{M}$ exerted the same effect than apelin $100 \mathrm{nM}$ on duodenal acetylcholine release (figure 2C). Conversely, apelin $1 \mu \mathrm{M}$ increased real-time duodenal NO release, indicating that this concentration of apelin is dependant of nNOS neurons to modulate its effect on duodenal contractions (figure 2D). This effect was antagonised by the APJ antagonist F13A (see online supplementary figure S3B).

To assess whether this switch is observed in in vivo conditions, we measured electrical duodenal activity in real time via a novel method using electrodes surrounding the duodenum linked to telemetric system (see online supplementary figure S3C). Apelin was injected directly into the duodenum via an intragastric catheter, whereby the electrical activity of the duodenum could be closely associated to mechanical activity. ${ }^{21}$ We found that apelin $100 \mathrm{nM}$ evoked a significant increase in duodenal electrical activity, while apelin $1 \mu \mathrm{M}$ decreased this activity (figure $2 \mathrm{E}$ ). All effects were abolished by APJ antagonist, demonstrating the specificity of apelin action (see online supplementary figure S3D). Thus, apelin is able to modify duodenal contractions via a mechanism involving the ENS (figure $2 \mathrm{~F}$ ).

Duodenum and jejunum are the two parts of the proximal intestine that both participate to the control of glucose absorption in various animal species. ${ }^{22}{ }^{23}$ Duodenum also expresses 


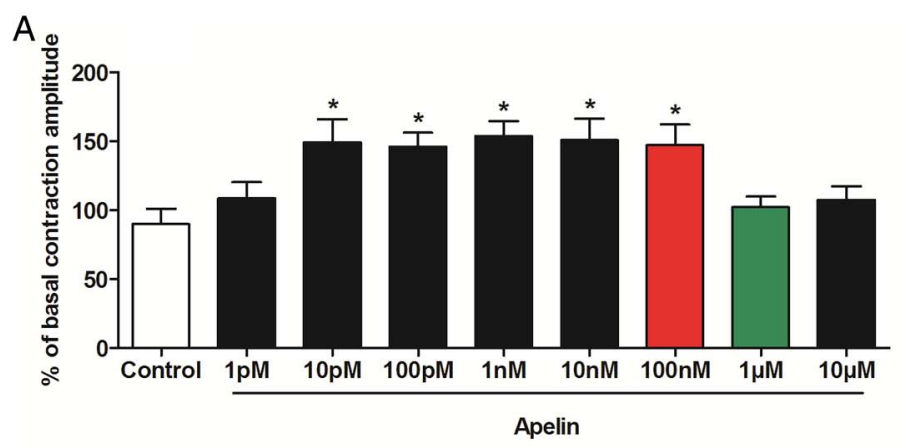

B
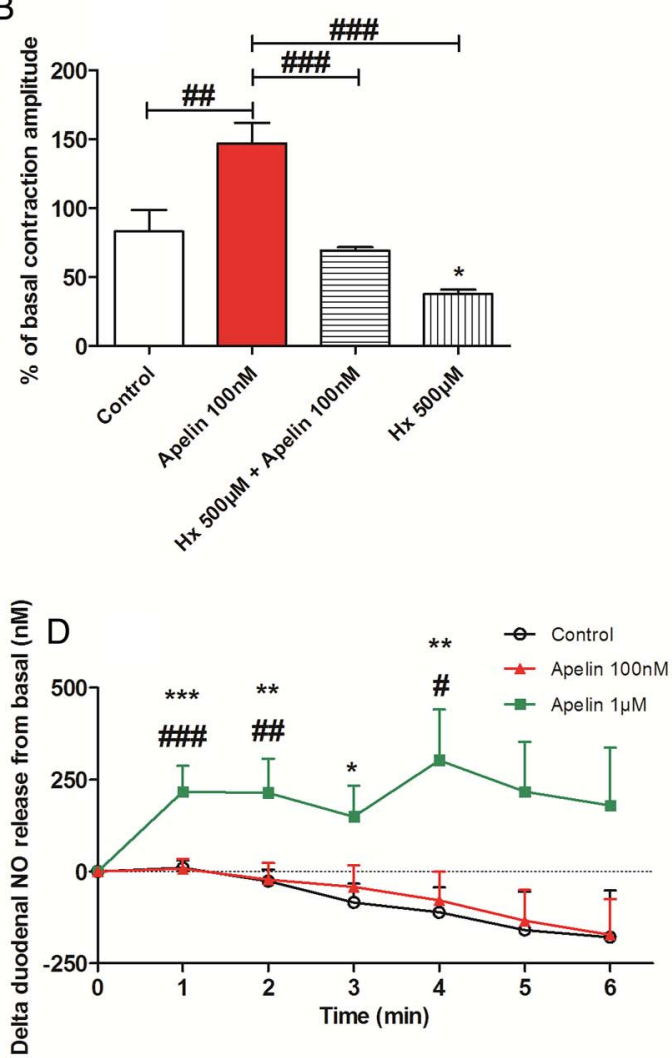

$\mathrm{F}$

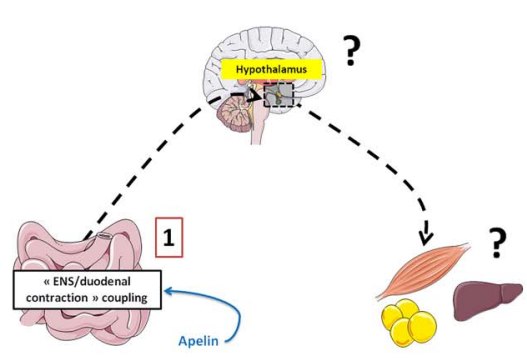

C

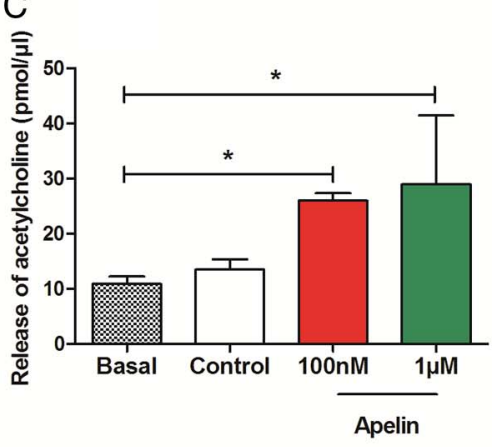

E

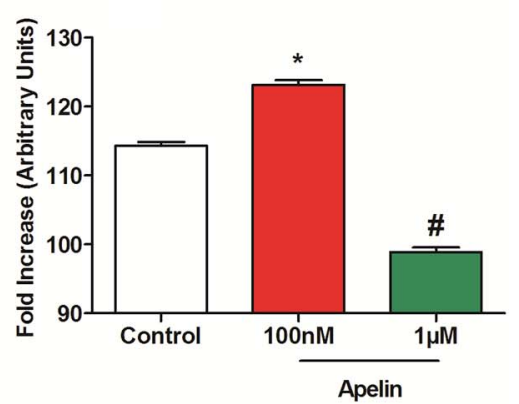

Figure 2 Apelin acts on the enteric nervous system to control duodenal contractions. (A) Ex vivo measurement of duodenal mechanical contraction amplitude in response to Krebs-Ringer solution (Control) or increasing concentrations of apelin ( $1 \mathrm{pM}$ to $10 \mu \mathrm{M}$ ). $\mathrm{n}=5-6$ per group. ${ }^{*} \mathrm{p}<0.05$ vs Control. (B) Ex vivo measurement of duodenal mechanical contraction amplitude in response to Krebs-Ringer (Control), apelin $100 \mathrm{nM}$, hexamethonium (Hx) $500 \mu \mathrm{M}$ alone or with apelin $100 \mathrm{nM}$. $n=5-6$ per group. ${ }^{*} \mathrm{p}<0.05$ vs Control. ${ }^{\#} \mathrm{p}<0.01,{ }^{\# \#} \mathrm{p}<0.001$. (C) Ex vivo measurement of duodenal acetylcholine release in basal condition and in response to Krebs-Ringer (Control), apelin $100 \mathrm{nM}$ or apelin $1 \mu \mathrm{M} . \mathrm{n}=4$ per group. ${ }^{*} p<0.05$. (D) Ex vivo measurement of duodenal nitric oxide (NO) release in response to Krebs-Ringer (Control), apelin $100 \mathrm{nM}$ or apelin $1 \mu \mathrm{M}$. $\mathrm{n}=5-8$ per group. ${ }^{*} \mathrm{p}<0.05,{ }^{* *} \mathrm{p}<0.01,{ }^{* * *} \mathrm{p}<0.001$ vs Control. ${ }^{*} \mathrm{p}<0.05,{ }^{\# \#} \mathrm{p}<0.01,{ }^{\# \#} \mathrm{p}<0.001$ vs apelin $100 \mathrm{nM}$. (E) In vivo measurement of duodenal electrical activity in response to $\mathrm{H}_{2} \mathrm{O}$ (Control), apelin $100 \mathrm{nM}$ or apelin $1 \mu \mathrm{M}$. $\mathrm{n}=4-5$ per group. ${ }^{*} \mathrm{p}<0.001$ vs Control and versus apelin $1 \mu \mathrm{M}$. ${ }^{\#}<<0.001$ vs Control and versus apelin $100 \mathrm{nM}$. (F) Schematic representation of the results: apelin modulates ENS activity causing a modification of duodenal contractions. 
intestinal glucose transporters and sensors. ${ }^{24-27}$ Previous studies have shown that stimulation of intestinal motility is associated with a rise in nutrient absorption. ${ }^{15}$ Our data show that apelin $100 \mathrm{nM}$ increased duodenal glucose absorption as opposed to apelin $1 \mu \mathrm{M}$ (figure 3A). No significant variation of apelin absorption was observed in our experimental model (figure $3 \mathrm{~B}$ ), but the level of apelin in the duodenum wall was significantly increased in apelin-treated duodenum fragments (see online supplementary figure S6A). In in vivo conditions, gut apelin instillation did not modify apelin concentrations in the portal vein (figure $3 \mathrm{C}$ ). These last results reinforce the concept of the specific action of apelin in ENS on duodenal wall.

\section{Duodenal apelin controls hypothalamic NO release}

$\mathrm{NO}$ is a central hypothalamic actor that controls glucose homeostasis. ${ }^{28}$ Moreover, alteration of intestinal glucose sensing linked to the gut-brain axis is associated to abnormal NO hypothalamic response in type 2 diabetes. ${ }^{6}$ Whether duodenal contractions are able to modify hypothalamic NO release is unknown. By using specific probes implanted in the hypothalamus of mice, we showed that decrease duodenal contraction by isoproterenol leads to an increase of hypothalamic NO release (see online supplementary figure S4A), demonstrating that intestinal motility is able to control hypothalamic activity. Using the same technique, gut administration of apelin $100 \mathrm{nM}$ decreased hypothalamic NO release while apelin $1 \mu \mathrm{M}$ allowed a return to basal NO levels (figure 4A). Importantly, this effect is blocked by the APJ antagonist, F13A (see online supplementary figure S4B, C). Moreover, blockade of nicotinic receptors with hexamethonium or destruction of afferent nerve endings with capsaicin abolishes the effect of gut apelin on hypothalamic NO release (figure 4B-D, see online supplementary figure S4D, E).

Here, our data prove the existence of a mode of communication between the gut and the brain, that is, duodenal contractility following apelin evoked signalling is able to modify hypothalamic NO release (figure 4E).

\section{Duodenal apelin controls muscle glucose utilisation via hypothalamic NO}

To measure the consequences of modification of duodenal contractions on glucose utilisation via the brain, we measured glucose entry in different tissues in response to oral gavage of apelin and radiolabeled glucose tracers in mice. Intragastric apelin $100 \mathrm{nM}$ decreased glucose entry in the muscle, but not in liver nor in adipose tissue (figure 5A-C). Similar to the effects observed on mechanical and electrical activities in the duodenum, apelin $1 \mu \mathrm{M}$ did not change basal glucose entry in the muscle. To further investigate whether brain release of $\mathrm{NO}$ is
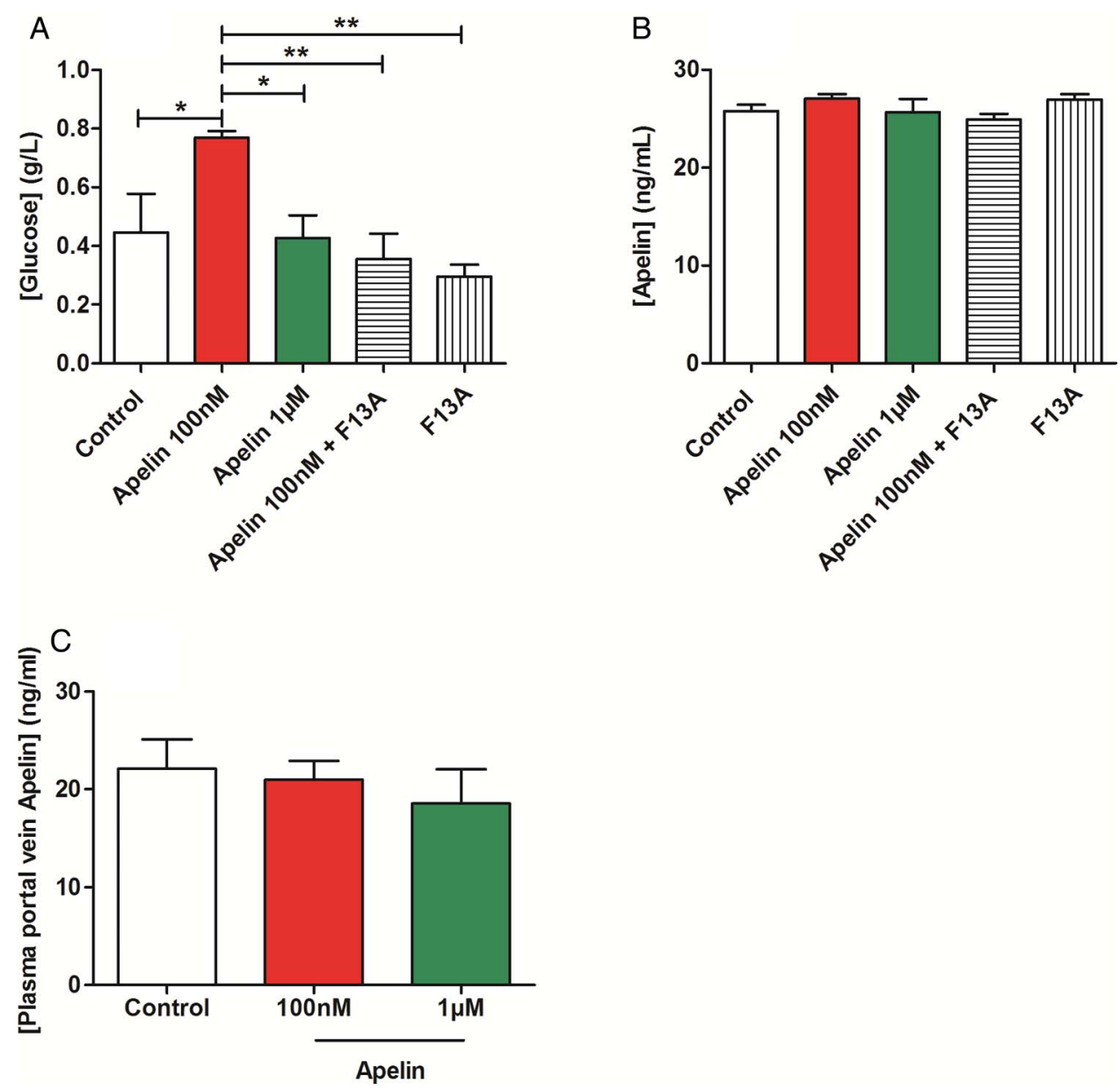

Figure 3 Apelin modulates duodenal glucose absorption. (A) Ex vivo glucose absorption in duodenal everted sacs in response to Krebs-Ringer (Control), apelin $100 \mathrm{nM}$, apelin $1 \mu \mathrm{M}, \mathrm{F} 13 \mathrm{~A}$ alone or with apelin $100 \mathrm{nM} . \mathrm{n}=5-7$ per group. ${ }^{*} p<0.05,{ }^{* *} p<0.01$. (B) Ex vivo apelin absorption in duodenal everted sacs in response to Krebs-Ringer (Control), apelin $100 \mathrm{nM}$ or $1 \mu \mathrm{M}$, and F13A alone or F13A plus apelin $100 \mathrm{nM}$. $\mathrm{n}=4-6 \mathrm{n}$ per group. (C) In vivo measurement of plasma apelin in portal vein, in response to oral gavage of $\mathrm{H}_{2} \mathrm{O}$ (Control), apelin $100 \mathrm{nM}$ or apelin $1 \mu \mathrm{M}$. $\mathrm{n}=5-6$ per group. 
mechanistically involved in the effect observed following intragastric apelin $1 \mu \mathrm{M}$ treatment on glucose muscle entry, we have blocked NO release in the brain by using intracerebroventricular injection of L-NMMA. We found that blocking NO release in the brain decreases glucose entry in the muscle (see online supplementary figure S5A-C), thereby showing the key role of brain $\mathrm{NO}$ on the apelin-gut to brain to muscle axis.

Together, our data show that apelin $100 \mathrm{nM}$ increases duodenal contractility and decreases muscle glucose utilisation (figure 5D) via a hypothalamic NO relay.
Chronic apelin gavage increases glucose tolerance via a decrease in duodenal motility

We hypothesised that a chronic stimulation by high level of apelin could provoke a decrease in duodenal contractility and could be associated with an improved glucose tolerance. To test whether chronic apelin treatment could modify basal duodenal contractility, and subsequently glucose utilisation, we measured basal mechanical activity in the duodenum of mice orally treated with apelin $(1 \mu \mathrm{M})$ for 1 week. Here, we show that chronic oral treatment with apelin increased apelin
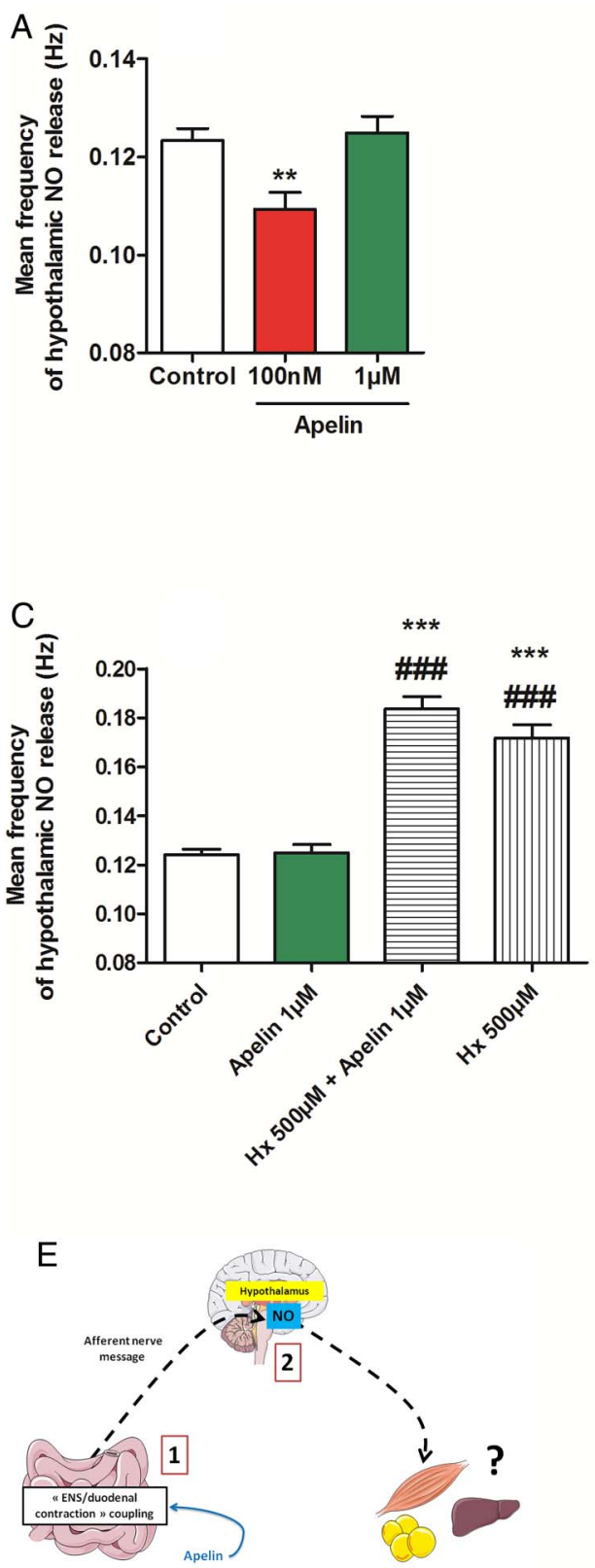

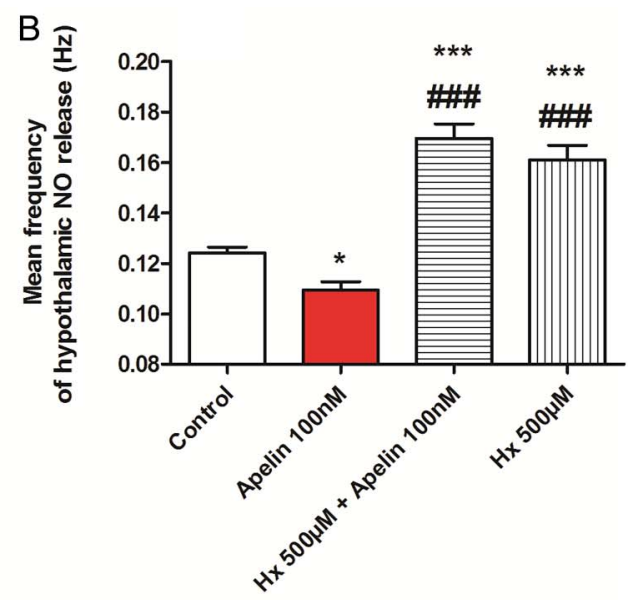

$\mathrm{D}$

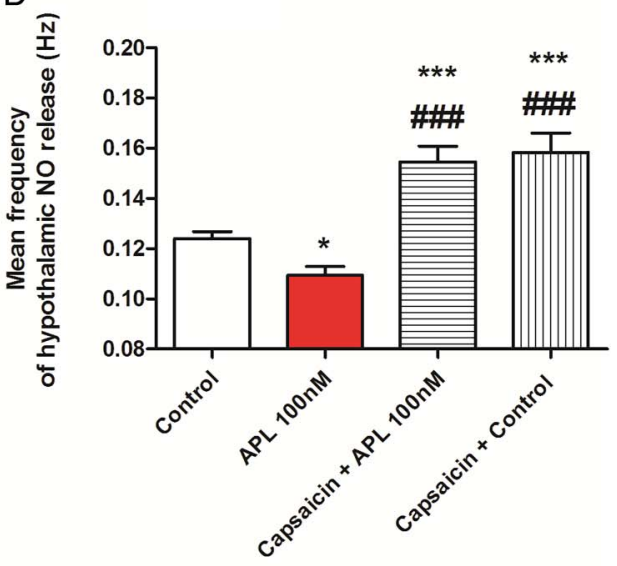

Figure 4 Duodenal apelin controls the gut motility-to-brain axis. (A) In vivo effect of intragastric perfusion of $\mathrm{H}_{2} \mathrm{O}$ (Control), apelin $100 \mathrm{nM}$ or apelin $1 \mu \mathrm{M}$, on nitric oxide (NO) hypothalamic release frequency. $n=5-7$ per group. ${ }^{*}{ }^{*} p<0.01$ vs Control. (B) In vivo effect of intragastric perfusion of $\mathrm{H}_{2} \mathrm{O}$ (Control), apelin $100 \mathrm{nM}$, hexamethonium (Hx) $500 \mu \mathrm{M}$ alone or with apelin $100 \mathrm{nM}$, on NO hypothalamic release frequency. $\mathrm{n}=5-8$ per group. ${ }^{*} p<0.01,{ }^{* *} p<0.001$ vs Control. ${ }^{\# \#} p<0.001$ vs apelin $100 \mathrm{nM}$. (C) In vivo effect of intragastric perfusion of $\mathrm{H}_{2} \mathrm{O}$ (Control), apelin $1 \mu \mathrm{M}, \mathrm{Hx}$ $500 \mu \mathrm{M}$ alone or with apelin $1 \mu \mathrm{M}$, on NO hypothalamic release frequency. $n=5-8$ per group. ${ }^{* *} p<0.001$ vs Control. ${ }^{* \#} p<0.001$ vs apelin $1 \mu \mathrm{M}$. (D) In vivo effect of intragastric perfusion of $\mathrm{H}_{2} \mathrm{O}$ (Control) and apelin $100 \mathrm{nM}$ in mice pretreated or not by capsaicin on NO hypothalamic release frequency. $n=4-7$ per group. ${ }^{*} p<0.01,{ }^{* * *} p<0.001$ vs Control. ${ }^{\# \#} p<0.001$ vs apelin $100 \mathrm{nM}$. (E) Schematic representation of the results: by modifying duodenal contractility, apelin modulates NO hypothalamic release. 

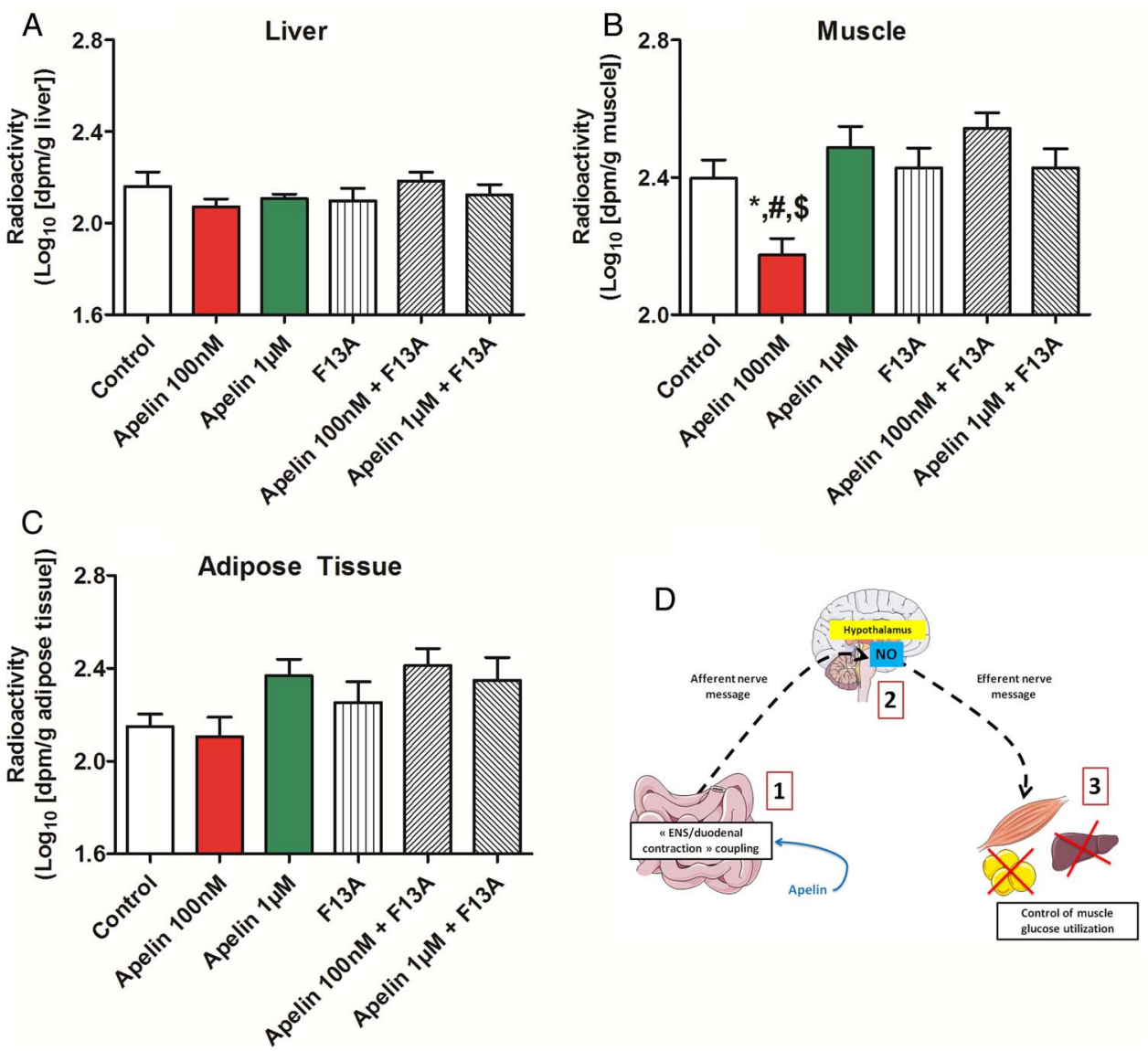

Figure 5 Duodenal apelin modulates muscle glucose utilisation. In vivo measurement of glucose entry in liver (A), muscle (B) and adipose tissue (C) in response to oral gavage of radiolabeled glucose in combination with $\mathrm{H}_{2} \mathrm{O}$ (Control), apelin $100 \mathrm{nM}$ alone or with $\mathrm{F} 13 \mathrm{~A}$, apelin $1 \mu \mathrm{M}$ alone or with F13A and F13A alone. $n=9-11$ per group. ${ }^{*} p<0.05$ vs Control and versus F13A. ${ }^{\#} p<0.01$ vs apelin $1 \mu M$ and versus apelin $1 \mu M+F 13 A$. ${ }_{p}<0.001$ vs apelin $100 \mathrm{nM}+\mathrm{F} 13 \mathrm{~A}$. (D) Schematic representation of the results: duodenal apelin controls muscle glucose utilisation via a hypothalamic relay.

concentration in the duodenum, but not in the jejunum and ileum; this observation reinforces the fact that the duodenum is a major target of transcytosed apelin (see online supplementary figure S6B). Chronic apelin treatment decreased basal duodenal contractility (figure 6A), increased glucose tolerance (figure 6B) and insulin release in response to glucose (figure 6C). Moreover, this was associated with an increase of glucose transporter type 4 (GLUT 4) mRNA expression (figure 6D) and glucose entry in the muscle (figure $6 \mathrm{E}$ ). No significant variation was observed regarding glucose entry in the liver and adipose tissue (see online supplementary figure S6C, D). This improvement of glucose tolerance was not associated to modification of body weight (control=25.2 $\pm 0.8 \mathrm{~g}$ vs apelin $1 \mu \mathrm{M}=23.3$ $\pm 0.9 \mathrm{~g})$.

\section{Apelin restores normal duodenal motility and improves glucose tolerance in obese/diabetic mice}

Similar to that observed in plasma and various tissues (eg, adipose tissue, muscle), we show that apelin mRNA level was significantly increased in the duodenum of HFD mice, but not in the jejunum and in the ileum (see online supplementary figure S7A). APJ mRNA expression was not modified in the proximal part of the intestine in HFD mice compared with control mice (see online supplementary figure S7B). First, we measured duodenal contraction in HFD mice in response to acute apelin $1 \mu \mathrm{M}$. We found that apelin $1 \mu \mathrm{M}$ counterbalanced the hypercontractility of duodenum generated by HFD treatment in ex vivo and in vivo conditions (figure 7A, B and online supplementary figure S7C). This effect was associated with an increase of duodenal NO release (figure $7 \mathrm{C}$ ) similar to that observed in normal mice (figure 2D). Then, the decrease of duodenal contractility is correlated with, first, a decrease intestinal glucose absorption (figure 7D), without effect on apelin absorption (figure 7E), and second, an increase in hypothalamic NO release in HFD apelin-treated mice (figure $7 \mathrm{~F}$ and online supplementary figure S7D). This modulation of gut-brain axis by apelin is associated with an improved glucose utilisation in muscle (figure 7G-I).

To test whether targeting the duodenal motility via apelin could be considered as a potential therapeutic target to treat type 2 diabetes, we measured the impact of a chronic ( 1 week) oral gavage of apelin in HFD mice. First, we found that chronic oral apelin administration improved fed glycaemia, fasted insulinemia and homeostasis model assessment insulin resistance of HFD mice (figure 8A). Second, chronic oral apelin treatment decreased basal duodenal motility (figure 8B) associated with an improved glucose tolerance (figure $8 \mathrm{C}$ ) and insulin resistance index (figure 8D) in HFD mice. Third, chronic apelin treatment increased GLUT4 mRNA expression in the muscle (figure 8E).

\section{DISCUSSION}

Circulating apelin is well recognised as a factor involved in the control of glucose homeostasis by modulating glucose utilisation acting directly on tissues (adipose tissue, muscle, intestine). In 


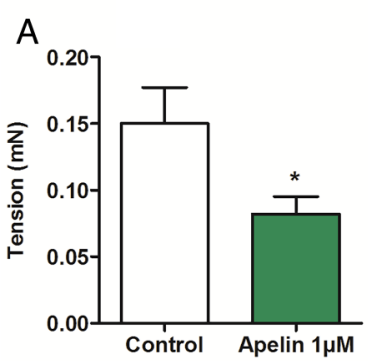

C
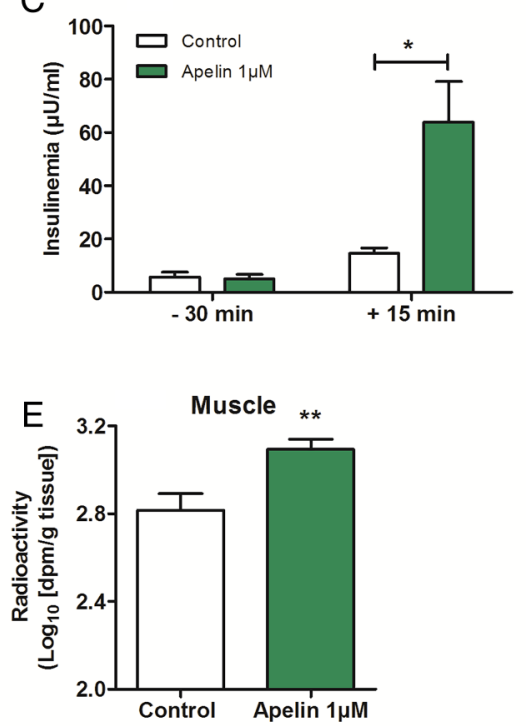
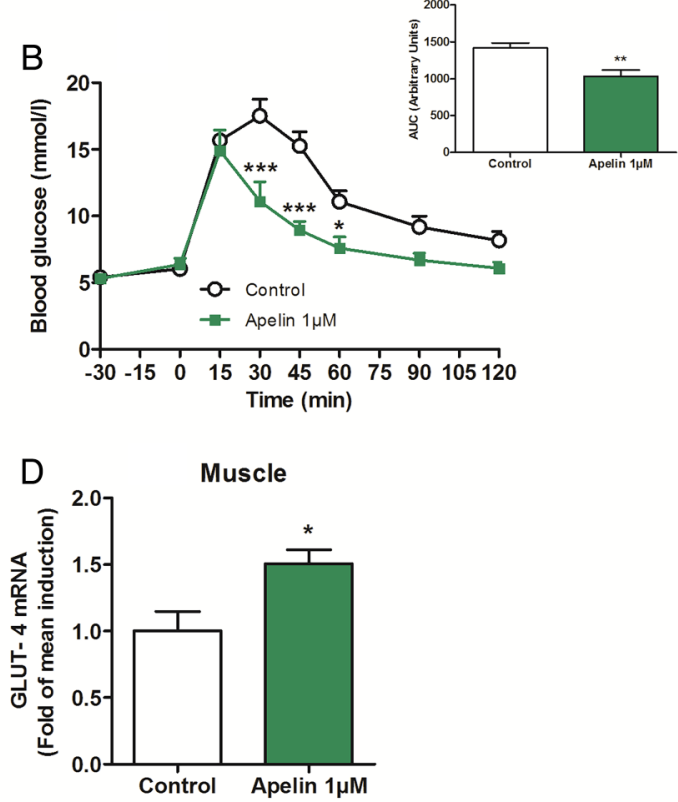

Figure 6 Chronic oral gavage of apelin $1 \mu \mathrm{M}$ increases glucose tolerance via a decrease in duodenal contractility. (A) Ex vivo measurement of duodenal mechanical contraction in mice orally gavaged for 1 week with $\mathrm{H}_{2} \mathrm{O}$ (Control) or apelin $1 \mu \mathrm{M}$. $\mathrm{n}=4-5$ per group. * $p<0.05$ vs Control. (B) Oral glucose tolerance test (OGTT) in $6 \mathrm{~h}$ fasted mice, orally gavaged for 1 week with $\mathrm{H}_{2} \mathrm{O}$ (Control) or apelin $1 \mu \mathrm{M}$. $\mathrm{n}=4-5 \mathrm{per}$ group. ${ }^{*} \mathrm{p}<0.05$, ${ }^{* *} \mathrm{p}<0.001$ vs Control. The adjacent graph represents the average area under the curve. (C) OGTT-associated insulinemia in $6 \mathrm{~h}$ fasted mice, orally gavaged for 1 week with $\mathrm{H}_{2} \mathrm{O}$ (Control) or apelin $1 \mu \mathrm{M}$. $\mathrm{n}=4-5$ per group. * $\mathrm{p}<0.05$. (D) Relative expression of glucose transporter type 4 mRNA in muscle of mice orally gavaged for 1 week with $\mathrm{H}_{2} \mathrm{O}$ (Control) or apelin $1 \mu \mathrm{M}$. $n=9-10$ per group. * $p<0.05$ vs Control. (E) In vivo measurement of glucose entry in muscle in response to oral gavage of radiolabeled glucose, in mice orally gavaged for 1 week with $\mathrm{H}_{2} \mathrm{O}(\mathrm{Control})$ or apelin $1 \mu \mathrm{M}$. $n=7$ per group. ${ }^{* *} p<0.01$ vs Control.

the brain, apelin exerts a dual effect depending on the nutritional state and its levels present in the hypothalamus. We have previously demonstrated that low dose of apelin administrated intracerebroventricularly improves glycaemia in fed mice. ${ }^{9}$ This effect could be linked to the slight and continuous increase in plasma apelin observed during the dark period (corresponding to the fed state). All these data suggest that a physiological rise of apelin in the whole body exerts beneficial effect on glucose metabolism as previously observed after an acute intravenous injection. ${ }^{10}$ Here, we show that apelin has a dual effect on ENS neurons similar to that observed in the CNS. In particular, we show that apelin induces modulations of duodenal contractility and subsequently modifies hypothalamic activity via variations of hypothalamic NO release to control muscle glucose utilisation. Thus, a parallel could be made between apelin's action on ENS and CNS neurons. In the brain, the effects of apelin on hypothalamic-releasing factors are different depending on the dose administered. Low dose of apelin stimulates NO release, while central high dose of apelin (which has deleterious action on glucose metabolism ${ }^{9}$ ) releases hydrogen peroxide instead of NO. ${ }^{29}$ In the duodenum, a similar differential effect on NO release is observed, suggesting that the recruitment of duodenal
nNOS neurons in response to apelin is dependent on the dose injected. In fact, apelin is able to stimulate acetylcholine release at $100 \mathrm{nM}$ and $1 \mu \mathrm{M}$, but this potential stimulatory effect of apelin on duodenal contractions is counterbalanced by the action of apelin $1 \mu \mathrm{M}$ on duodenal NO release. For these reasons, apelin $1 \mu \mathrm{M}$ effect on duodenal contractions is similar to control in normal mice. This hypothesis is supported by data obtained in HFD mice since apelin $1 \mu \mathrm{M}$ stimulates duodenal NO release to decrease duodenal hypercontractility. We cannot totally rule out the possibility that apelin $1 \mu \mathrm{M}$ exerts downregulation of APJ expression in our experimental models similar to that observed in the hypothalamus of obese rats, ${ }^{30}$ but our data tend to limit this possibility. First, we show that apelin mRNA expression is increased in the duodenum of HFD mice, without modification of APJ mRNA expression. This result supports data from the literature, suggesting that peripheral increase expression of apelin could represent an adaptive pathway to counterbalance insulin resistance. ${ }^{8}$ Second, duodenal fragments release $\mathrm{NO}$ in response to apelin $1 \mu \mathrm{M}$, but not $100 \mathrm{nM}$, reinforcing the existence of two different molecular actors to respectively block and stimulate duodenal contractions. Then, our data suggest that ENS neurons are potential targets of 
A

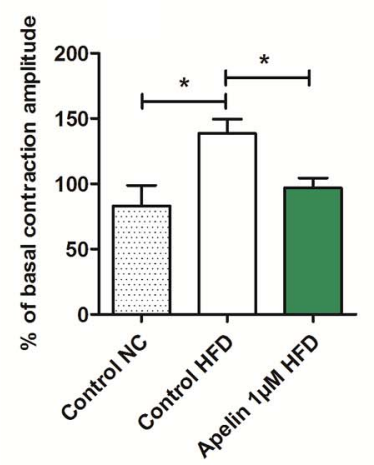

$\mathrm{D}$
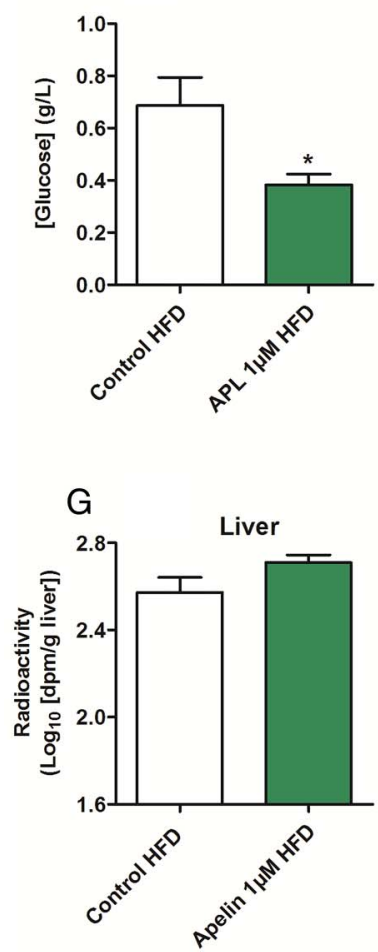

B

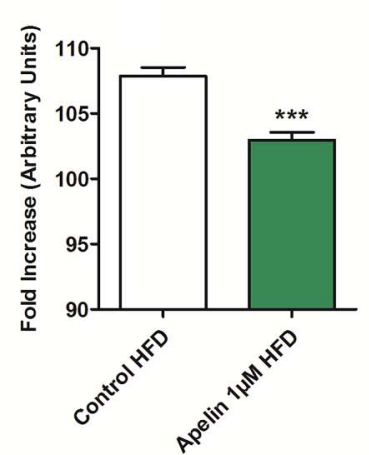

$\mathrm{E}$
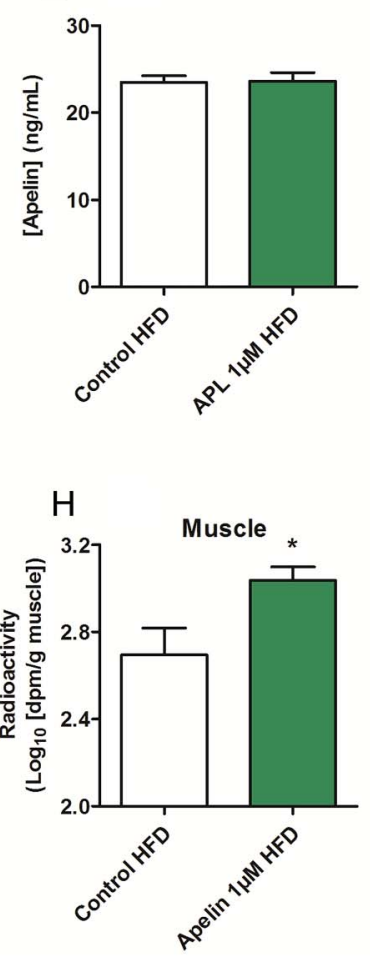
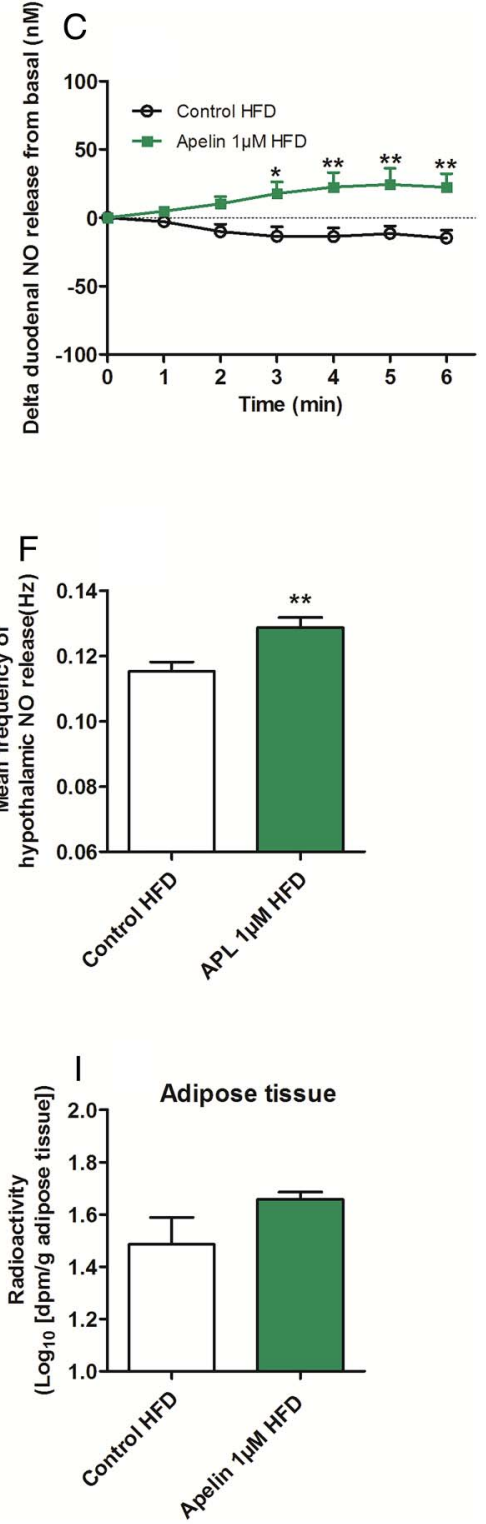

Figure 7 Apelin $1 \mu \mathrm{M}$ decreases duodenal contractility and improves glucose utilisation in obese/diabetic mice. (A) Ex vivo measurement of duodenal mechanical contraction amplitude in response to Krebs-Ringer (Control) (in normal chow or high-fat diet (HFD) mice) and apelin $1 \mu M$ (in HFD mice). $n=5-6$ per group. ${ }^{*} p<0.05$. (B) In vivo measurement of duodenal electrical activity in response to $\mathrm{H}_{2} \mathrm{O}$ (Control HFD) and apelin $1 \mu \mathrm{M}$. $\mathrm{n}=4-7$ per group. ${ }^{* *} \mathrm{p}<0,001$ vs Control HFD. (C) Duodenal nitric oxide (NO) release in response to Krebs-Ringer (Control HFD) or apelin $1 \mu \mathrm{M}$. $n=4-7$ per group. ${ }^{*} p<0.05,{ }^{* *} p<0.01$ vs Control HFD. (D) Ex vivo glucose absorption in duodenal everted sacs in response to Krebs-Ringer (Control HFD) and apelin $1 \mu \mathrm{M}$. $n=4-6$ per group. * $p<0.05$ vs Control HFD. (E) Ex vivo apelin absorption in duodenal everted sacs in response to KrebsRinger (Control HFD) and apelin $1 \mu \mathrm{M}$. $n=6$ per group. (F) In vivo effect of intragastric perfusion of $\mathrm{H}_{2} \mathrm{O}$ (Control HFD) and apelin $1 \mu \mathrm{M}$ on NO hypothalamic release frequency. $n=6$ per group. ${ }^{* *} p<0.01$ vs Control HFD. In vivo measurement of glucose entry in liver $(G)$, muscle $(H)$ and adipose tissue (I) in response to oral gavage of radioactive glucose in combination with $\mathrm{H}_{2} \mathrm{O}$ (Control HFD) and apelin. $n=4-6$ per group. ${ }^{*} p<0.05$ vs Control HFD.

apelin action on duodenal motility since (1) apelin modulates duodenal acetylcholine and NO release, (2) apelin stimulatory effect is blocked by hexamethonium and (3) apelin receptor APJ is expressed on ENS neurons. As apelin receptor APJ is expressed in the intestinal wall, we can speculate that apelin may also exert effects on other intestinal cells. Thus, we cannot exclude the potential existence of a direct action of apelin on intestinal smooth muscles.

In the present study, we proposed a new and physiologically relevant mode of communication between the gut and the brain that controls glucose utilisation. Specifically, our data suggest that peripheral glucose utilisation can be controlled by duodenal contractility via a hypothalamic relay. In physiological conditions, our data suggest that apelin exerts differential effect on ENS neurons during digestion. Therefore, we propose the following model, at the beginning of food intake, apelin has a stimulatory effect (1) on intestinal glucose absorption via actions on GLUT2 translocation ${ }^{11}$ and (2) on duodenal motility. This stimulatory effect on intestinal contraction leads to a hypothalamic NO blockade that decreases glucose utilisation in tissues in order to avoid a potential hypoglycaemia. At the end of the meal, high levels of apelin target nNOS neurons to (1) slow down duodenal waves and stop glucose absorption, (2) restore hypothalamic NO release and (3) increase muscle 


A
\begin{tabular}{|c|c|c|c|c|c|}
\hline Parameters & NC & HFD & $\begin{array}{c}\text { HFD } \\
\text { APL } 1 \mu M\end{array}$ & $\begin{array}{c}\text { Statistics } \\
(\text { NC vs HFD) }\end{array}$ & $\begin{array}{c}\text { Statistics } \\
\text { (HFD vs } \\
\text { HFD APL } 1 \mu \text { M) }\end{array}$ \\
\hline Weight (g) & $25,23 \pm 0,87$ & $46,23 \pm 0,91$ & $45,70 \pm 0,47$ & $* * *$ & ns \\
\hline $\begin{array}{c}\text { Fasted glucose } \\
\text { (mmol/L) }\end{array}$ & $5,40 \pm 0,20$ & $12,72 \pm 0,86$ & $11,14 \pm 0,30$ & $* * *$ & ns \\
\hline Fed glucose (mmol/L) & $8,56 \pm 0,21$ & $11,34 \pm 0,35$ & $9,66 \pm 0,65$ & ${ }^{* * *}$ & $\mathrm{p}=0,0588$ \\
\hline Fasted insulin (mU/L) & $5,82 \pm 1,85$ & $65,41 \pm 10,93$ & $29,43 \pm 9,01$ & ${ }^{* * *}$ & ${ }^{* *}$ \\
\hline HOMA-IR & $1,37 \pm 0,43$ & $41,56 \pm 7,29$ & $15,05 \pm 4,86$ & ${ }^{* * *}$ & $*$ \\
\hline
\end{tabular}
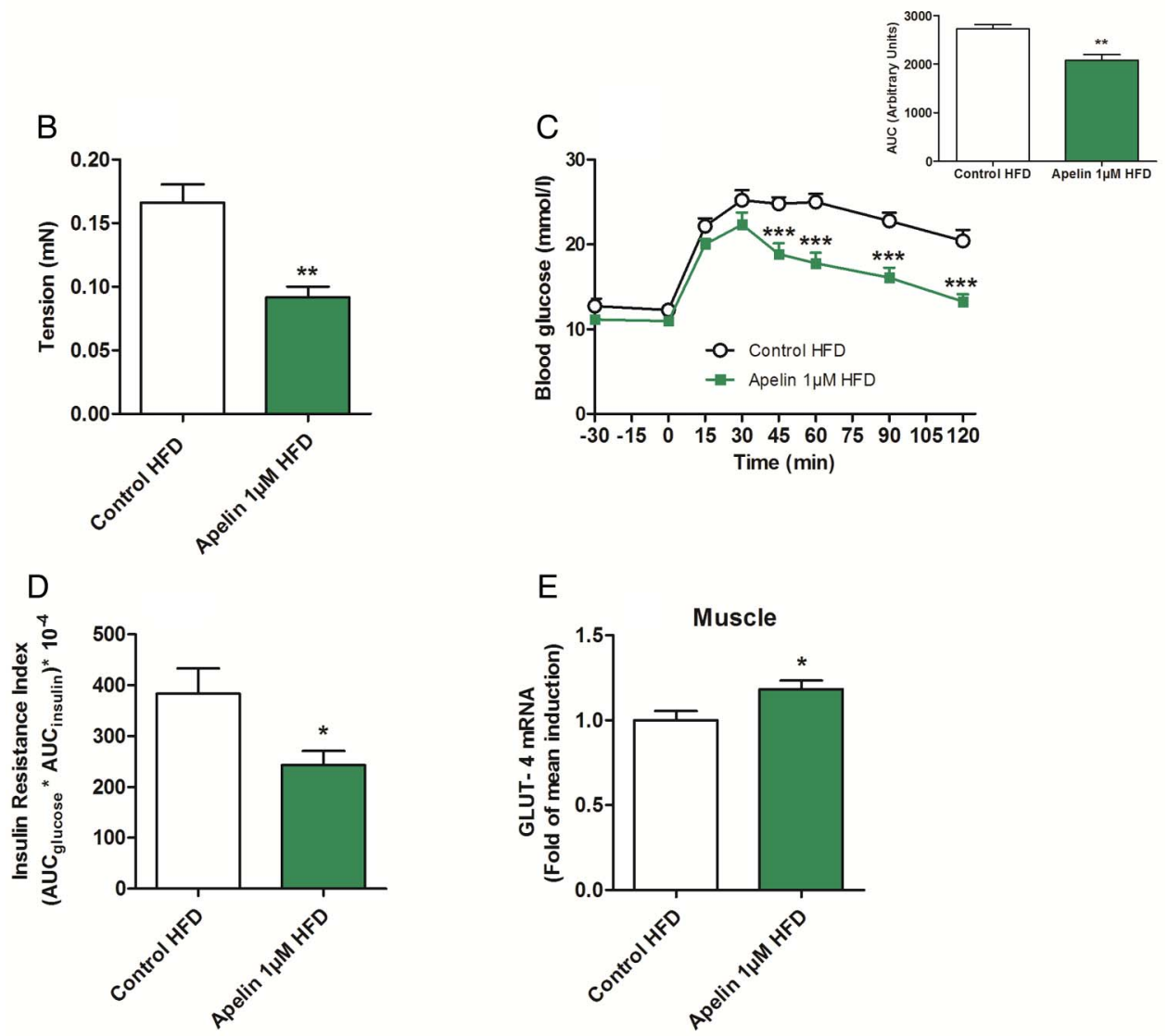

Figure 8 Apelin $1 \mu \mathrm{M}$ treatment increases glucose tolerance via a decrease in duodenal contractility. (A) Effects of high-fat diet (HFD) on metabolic parameters in mice orally gavaged for 1 week with $\mathrm{H}_{2} \mathrm{O}$ (Control HFD) or apelin $1 \mu \mathrm{M}$. $\mathrm{n}=6$ per group. (B) Ex vivo measurement of duodenal mechanical contraction in HFD mice orally gavaged for 1 week with $\mathrm{H}_{2} \mathrm{O}$ (Control HFD) or apelin $1 \mu \mathrm{M}$. $n=6$ per group. ${ }^{* *} p<0.01$ vs Control HFD. (C) Oral glucose tolerance test (OGTT) in $6 \mathrm{~h}$ fasted HFD mice, orally gavaged for 1 week with $\mathrm{H}_{2} \mathrm{O}$ (Control HFD) or apelin $1 \mu M$. $\mathrm{n}=6$ per group. ${ }^{* *} p<0.01,{ }^{* *} p<0.001$ vs Control HFD. The adjacent graph represents the average area under the curve (AUC). (D) OGTT-associated insulin resistance index in $6 \mathrm{~h}$ fasted HFD mice, orally gavaged for 1 week with $\mathrm{H}_{2} \mathrm{O}$ (Control HFD) or apelin $1 \mu \mathrm{M}$. $\mathrm{n}=6$ per group. * $\mathrm{p}<0.05$ vs Control HFD. (E) Relative expression of glucose transporter type 4 (GLUT-4) mRNA in muscle of HFD mice orally gavaged for 1 week with $\mathrm{H}_{2} \mathrm{O}$ (Control HFD) or apelin $1 \mu \mathrm{M}$. $\mathrm{n}=9-10$ per group. ${ }^{*} \mathrm{p}<0.05$ vs Control. HOMA-IR, homeostasis model assessment insulin resistance; NC, normal chow.

glucose utilisation. The association between central NO and peripheral glucose utilisation has been previously demonstrated by our group since an increase in hypothalamic NO release stimulates peripheral vascular flux, leading to glucose entry in muscle. $^{28}$

Such regulations of glucose homeostasis implicating the gutbrain axis and an intestinal sensor during the digestion have clearly been established. We have previously demonstrated that intestinal glucose sensing during hyperglycaemia generates an increase in hepatic glycogenesis to prevent the next period of fasting via a central relay. ${ }^{17}$ This phenomenon implicates a hypothalamic GLP-1 pathway; GLP-1 is, similar to apelin, an intestinal hormone present in the brain and playing an important role as a neurotransmitter. ${ }^{2}$ All these data implicating intestinal hormonal sensing and mechanosensing highlight the importance of the 'gut-to-brain-to-peripheral' axis in the control of glucose metabolism.

Type 2 diabetes is associated with insulin resistance. We have previously demonstrated that alteration of intestinal glucose sensing participates to the insulin resistance state observed in obese/diabetic mice. ${ }^{2}{ }^{6}$ In fact, intestinal inflammation and oxidative stress are associated with abnormal glucose sensing, leading to aberrant hypothalamic NO release. ${ }^{6}$ This alteration of glucose detection could be associated with disturbance of mechanodetection. Accordingly, it has been shown that type 2 diabetes is associated with a decrease of nNOS neuronal 
population in the ENS ${ }^{12}$ generating an increase of intestinal motility. ${ }^{12} 31$ Intestinal hypermotility could first induce an increase in duodenal glucose absorption, leading to fed hyperglycaemia, and second, according to our results, it may cause insulin resistance via the brain. Here, we have shown that high levels of apelin are able to restore basal duodenal contraction, specifically in the proximal portion of the intestine in normal and obese/diabetic mice. Targeting the ENS neurons to modulate gut contraction via apelin or another molecular target represents an invaluable tool to decrease duodenal contraction, which could have a real potential interest for new therapeutic strategies to treat type 2 diabetes comorbidities such as hyperglycaemia and insulin resistance. Our data support this hypothesis since chronic oral apelin ameliorates glucose tolerance in HFD mice.

Numerous factors could reach the ENS neurons to modulate their activities. Some of them could have a plasma origin, and others could act from the intestinal lumen to the ENS via transcytosis as in the case of leptin. ${ }^{18}$ Apelin is known to be released in the lumen by enterocytes in response to glucose to aid glucose absorption. ${ }^{11}$ In this study, we show that transcytosis of apelin in the duodenum controls glucose absorption via an ENS-dependent mechanism. Therefore, our data highlight a novel mode of regulation of glucose homeostasis involving an intestinal factor. The importance of gut hormones such as GLP-1 in the control of glucose metabolism is well established ${ }^{32}$ and represents an innovative approach to treat metabolic disorders. In addition to their direct effects on insulin-sensitive tissues, the potential actions of these factors on 'ENS/contraction-to brain-to peripheral' axis could bring new possibilities and combinations to improve diabetic state. Actually, type 2 diabetic strategies aim to target GLP-1, which is released by L-cells located in the ileum. Another originality of our work is to target the first site of nutrients detection, that is, the duodenum, which is now considered, like jejunum, ${ }^{7}$ as a future target to restore glucose homeostasis during diabetes. ${ }^{5}$ Nutrient sensing, which is largely altered in type 2 diabetes, ${ }^{5}$ could be compensated by modulation of duodenal contraction as a therapeutic action to suppress hyperglycaemia. This hypothesis is supported by the work of Sandoval et al, ${ }^{33}$ which demonstrates that internal mechanical manipulation of the duodenum after fasting generates gut hormones release. We speculate that modulation of intestinal contractions by apelin or other factors could involve a hormonal-mediated signal in addition to the capsaicinsensitive afferent nervous signal demonstrated in the present study.

In conclusion, we provide evidence that interactions between the ENS, duodenal contractility and the brain are of crucial importance to control glucose utilisation in physiological conditions. Moreover, we demonstrate that intestinal apelin is a key actor playing a major role in this system. Besides, numerous possibilities exist in terms of (1) potential other molecular factors (eg, hormones) targeting this system or (2) potential effects on physiological functions (eg, food intake, lipid metabolism); our study illustrates the mechanisms of action of intestinal apelin and its impact on glucose homeostasis. Future explorations are warranted to demonstrate the role of this axis to treat metabolic diseases in humans.

\footnotetext{
Author affiliations

${ }^{1}$ Institut National de la Santé et de la Recherche Médicale (INSERM), U1048, Institut des Maladies Métaboliques et Cardiovasculaires (I2MC), Toulouse Cedex 4, France ${ }^{2}$ NeuroMicrobiota, European Associated Laboratory (EAL) INSERM/UCL

${ }^{3}$ Université Paul Sabatier, Toulouse, France

${ }^{4}$ Université Catholique de Louvain (UCL), Louvain Drug Research Institute, LDRI, Metabolism and Nutrition research group, WELBIO (Walloon Excellence in Life sciences and BIOtechnology), Brussels, Belgium
}

${ }^{5}$ Visceral Pain Group, Centre for Nutrition and Gastrointestinal Diseases, Discipline of Medicine, University of Adelaide, South Australia Health and Medical Research Institute (SAHMRI), Adelaide, South Australia, Australia

${ }^{6}$ Department of Gastroenterology and Hepatology, Royal Adelaide Hospital, Adelaide, South Australia, Australia

${ }^{7}$ Discipline of Physiology, Faculty of Health Sciences, The University of Adelaide, Adelaide, South Australia, Australia

${ }^{8}$ Institut National de la Santé et de la Recherche Médicale (INSERM), U1037, Centre de Recherches en Cancérologie de Toulouse (CRCT), CHU Rangueil, Toulouse, Cedex 4, France

${ }^{9}$ Institut National de la Santé et de la Recherche Médicale (INSERM), U1043, Centre de Physiopathologie de Toulouse Purpan (CPTP), CHU Purpan, Toulouse, Cedex 03, France

Acknowledgements We thank Sophie Allart and Astrid Canivet for technical assistance at the cellular imaging facility of INSERM UMR 1043, Toulouse, the Service Phénotypage UMS US006/INSERM for telemetric measurements, and Simon Nicolas and Isabelle Castan-Laurell for helpful discussion.

Contributors $A F$ and $A D$ contributed equally. $A F, A D, T D, A M, S M B, J C, S L-G$, $B M, A C, A L, P R, N C, P D C$ and $C K$ acquired data. $A F, A D, P D C$ and $C K$ have performed the study concept and design. $A F, A D, P D C$ and $C K$ analysed and interpreted the data. AF, AD, TD, SMB, JC, NC, NV, PV, PDC and CK wrote the manuscript.

Funding This work was supported by the Fonds de la Recherche ScientifiqueFNRS for the FRFS-WELBIO under grant: WELBIO-CR-2012S-02R. This work was supported in part by the Funds InBev-Baillet Latour (Grant for Medical Research 2015). The authors thank the Societe Francaise de Nutrition (SFN) and the Fondation Recherche Médicale (FRM) (Grant ING20150532586) for financia support. PDC is the recipient of grants from FNRS (convention J.0084.15, convention 3.4579.11), PDR (Projet de Recherche, convention: T.0138.14), FRM (Fondation Recherche Medicale) and ARC (Action de Recherche Concertée-Communauté française de Belgique convention: 12/17-047). PDC is also a recipient of an ERC Starting Grant 2013 (European Research Council, Starting grant 336452-ENIGMO). NV is recipient of an ERC (Consolidator Grant, PIPE). SMB is an NHMRC Australia R. D Wright Biomedical Fellow.

\section{Competing interests None declared.}

Ethics approval Experiments were conducted according to the European Community regulations concerning the protection of experimental animals and were approved by the local Animal Care and Use Committee.

Provenance and peer review Not commissioned; externally peer reviewed.

Open Access This is an Open Access article distributed in accordance with the Creative Commons Attribution Non Commercial (CC BY-NC 4.0) license, which permits others to distribute, remix, adapt, build upon this work non-commercially, and license their derivative works on different terms, provided the original work is properly cited and the use is non-commercial. See: http://creativecommons.org/ licenses/by-nc/4.0/

\section{REFERENCES}

1 Blouet C, Schwartz GJ. Duodenal lipid sensing activates vagal afferents to regulate non-shivering brown fat thermogenesis in rats. PLOS ONE 2012;7:e51898.

2 Knauf C, Cani PD, Kim DH, et al. Role of central nervous system glucagon-like Peptide-1 receptors in enteric glucose sensing. Diabetes 2008:57:2603-12.

3 Clapham JC. Central control of thermogenesis. Neuropharmacology 2012;63:111-23.

4 Coll AP, Yeo GS. The hypothalamus and metabolism: integrating signals to contro energy and glucose homeostasis. Curr Opin Pharmacol 2013;13:970-6.

5 Breen DM, Rasmussen BA, Cote CD, et al. Nutrient-sensing mechanisms in the gut as therapeutic targets for diabetes. Diabetes 2013;62:3005-13.

6 Duparc T, Naslain D, Colom A, et al. Jejunum inflammation in obese and diabetic mice impairs enteric glucose detection and modifies nitric oxide release in the hypothalamus. Antioxid Redox Signal 2011;14:415-23.

7 Rasmussen BA, Breen DM, Duca FA, et al. Jejunal leptin-PI3K signaling lowers glucose production. Cell Metab 2014;19:155-61.

8 Castan-Laurell I, Dray C, Knauf C, et al. Apelin, a promising target for type 2 diabetes treatment? Trends Endocrinol Metab 2012;23:234-41.

9 Duparc T, Colom A, Cani PD, et al. Central apelin controls glucose homeostasis via a nitric oxide-dependent pathway in mice. Antioxid Redox Signal 2011;15:1477-96.

10 Dray C, Knauf C, Daviaud D, et al. Apelin stimulates glucose utilization in normal and obese insulin-resistant mice. Cell Metab 2008:8:437-45.

11 Dray C, Sakar Y, Vinel C, et al. The intestinal glucose-apelin cycle controls carbohydrate absorption in mice. Gastroenterology 2013;144:771-80.

12 Chandrasekharan B, Srinivasan S. Diabetes and the enteric nervous system. Neurogastroenterol Motil 2007;19:951-60. 
13 Reichardt F, Krueger D, Schemann M. Leptin excites enteric neurons of guinea-pig submucous and myenteric plexus. Neurogastroenterol Motil 2011;23:e165-70.

14 Bjerknes $M$, Cheng $H$. Modulation of specific intestinal epithelial progenitors by enteric neurons. Proc Natl Acad Sci USA 2001;98:12497-502.

15 Sababi M, Bengtsson UH. Enhanced intestinal motility influences absorption in anaesthetized rat. Acta Physio/ Scand 2001;172:115-22.

16 Masri B, Salahpour A, Didriksen M, et al. Antagonism of dopamine D2 receptor/ beta-arrestin 2 interaction is a common property of clinically effective antipsychotics. Proc Natl Acad Sci USA 2008;105:13656-61.

17 Knauf C, Cani PD, Perrin C, et al. Brain glucagon-like peptide-1 increases insulin secretion and muscle insulin resistance to favor hepatic glycogen storage. J Clin Invest 2005; 115:3554-63.

18 Cammisotto PG, Gingras D, Bendayan M. Transcytosis of gastric leptin through the rat duodenal mucosa. Am J Physiol Gastrointest Liver Physiol 2007;293: G773-9.

19 Zizzo MG, Mule F, Serio R. Duodenal contractile activity in dystrophic (mdx) mice: reduction of nitric oxide influence. Neurogastroenterol Motil 2003;15:559-65.

20 Zizzo MG, Mule F, Serio R. Inhibitory responses to exogenous adenosine in murine proximal and distal colon. Br J Pharmacol 2006;148:956-63.

21 Postorino A, Mancinelli R, Racanicchi C, et al. Spontaneous electromechanical activity in the rat duodenum in vitro. Arch Int Physiol Biochim 1990;98:35-40.

22 Riesenfeld G, Sklan D, Bar A, et al. Glucose absorption and starch digestion in the intestine of the chicken. J Nutr 1980;110:117-21.

23 Camacho RC, Denny JC, Pencek RR, et al. Portal venous hyperinsulinemia does not stimulate gut glucose absorption in the conscious dog. Metabolism 2004:53:1290-5
24 Kim HR, Park SW, Cho HJ, et al. Comparative gene expression profiles of intestinal transporters in mice, rats and humans. Pharmacol Res 2007;56:224-36.

25 Lohrenz AK, Duske K, Schonhusen U, et al. Glucose transporters and enzymes related to glucose synthesis in small intestinal mucosa of mid-lactation dairy cows fed 2 levels of starch. J Dairy Sci 2011;94:4546-55.

26 Pfannkuche H, Gabel G. Glucose, epithelium, and enteric nervous system: dialogue in the dark. J Anim Physiol Anim Nutr (Berl) 2009;93:277-86.

27 Shirazi-Beechey SP, Moran AW, Batchelor DJ, et al. Glucose sensing and signalling; regulation of intestinal glucose transport. Proc Nutr Soc 2011;70:185-93.

28 Cabou C, Cani PD, Campistron G, et al. Central insulin regulates heart rate and arterial blood flow: an endothelial nitric oxide synthase-dependent mechanism altered during diabetes. Diabetes 2007;56:2872-7.

29 Drougard A, Duparc T, Brenachot $X$, et al. Hypothalamic apelin/reactive oxygen species signaling controls hepatic glucose metabolism in the onset of diabetes. Antioxid Redox Signal 2014;20:557-73.

30 Clarke KJ, Whitaker KW, Reyes TM. Diminished metabolic responses to centrally-administered apelin-13 in diet-induced obese rats fed a high-fat diet. J Neuroendocrinol 2009;21:83-9.

31 Carrier GO, Aronstam RS. Increased muscarinic responsiveness and decreased muscarinic receptor content in ileal smooth muscle in diabetes. J Pharmacol Exp Ther 1990;254:445-9.

32 Everard A, Cani PD. Gut microbiota and GLP-1. Rev Endocr Metab Disord 2014;15:189-96.

33 Sandoval D, Dunki-Jacobs A, Sorrell J, et al. Impact of intestinal electrical stimulation on nutrient-induced GLP-1 secretion in vivo. Neurogastroenterol Motil 2013;25:700-5 